\title{
Gas-Assisted Focused Ion Beam Fabrication of Gold Nanoelectrode Arrays in Electron-Beam Evaporated Alumina Films for Biosensing Applications
}

\author{
Napat Trirol $^{1,3, *}$, Papot Jaroenapibal ${ }^{2,4}$, and Roderic Beresford ${ }^{3}$ \\ ${ }^{1}$ Department of Electrical Engineering, Faculty of Engineering, Khon Kaen University, \\ Khon Kaen 40002, Thailand. \\ "Corresponding author's e-mail address: napat@elec.kku.ac.th, napatr@kku.ac.th \\ ${ }^{2}$ Department of Industrial Engineering, Faculty of Engineering, Khon Kaen University, \\ Khon Kaen 40002, Thailand. \\ ${ }^{3}$ Division of Engineering, Brown University, 182 Hope Street, Providence, RI 02912, USA. \\ ${ }^{4}$ Department of Materials Science and Engineering, University of Pennsylvania, 3231 Walnut Street, \\ Philadelphia, PA 19104, USA.
}

\begin{abstract}
:
This work reports the fabrication details used to prepare gold nanoelectrode arrays in alumina substrates. The fabrication steps include electron-beam evaporation of $20-\mathrm{nm} \mathrm{Ti}$ and $300-\mathrm{nm}$ thick Au for the electrode platform on a glass substrate, followed by electron-beam evaporation of $800-\mathrm{nm}$ thick $\mathrm{Al}_{2} \mathrm{O}_{3}$ as a passivation layer. $\mathrm{I}_{2}$-assisted focused ion beam (FIB) milling is employed to create highaspect-ratio pores in the alumina films. The final pore size of $60-120 \mathrm{~nm}$ is achieved by ion beam sculpting after the initial milling process. The nanopores are then filled with Au via electrodeposition to obtain a nanoelectrode structure. Cyclic voltammetry (CV) responses of a standard redox species exhibit a nonclassical behavior of which a well-defined steady-state limiting current plateau is not observed.
\end{abstract}

Key words: nanoelectrode arrays, focused ion beam, aluminum oxide, cyclic voltammetry

\section{Introduction}

An extremely low-level signal from a bioreaction process can be acquired into a measurable electrical signal through highly efficient electrodes. Electrodes of nanometer size possess unique properties which create the possibility of direct electronic transduction of bio-signals. Some studies have shown that the electrochemical behavior of nanoscale electrodes deviates significantly from the predictions of traditional theories. The departure is found to be dependent on the electrode size as it approaches values of molecular dimensions. Those deviations are, for example, the high electric field generated near the electrode surface that causes an enhancement or inhibition of the flux of the electroactive species to the electrode surface [1], the departure of the diffusion current from that under the classical diffusion control theory [2], and the shift of the half-wave potential due to the increase in the current density [3]. Such deviations can be observed from the shape of the voltammetric response curves.
As the electrode size decreases, radial diffusion becomes dominant and the depletion layer thickness approaches the size of the electrode dimension [4]. Since the measured current density is proportional to the inverse of the depletion layer thickness, smaller electrode gives higher measured current density due to thinner diffusion layer. This phenomenon is called enhanced mass transport [5]. Besides the enhancement of mass transport, the electric field and charge within the near-electrode layers become significant influences on the mass transport and electron transfer kinetics at the nanoscale electrodes. For electrodes having a radius less than $100 \mathrm{~nm}$, the electrical double layer, a region close to the electrode surface where a high electric field exists, begins to occupy an appreciable fraction of the depletion layer. The electrostatic force within the double layer will accelerate or retard the flux of the charged species to the electrode surface. Therefore, the migration resulting from the high electric field within the double layer will be added to the mass transport equation. The enhanced electric field will enable significant improvement in electronic transduction of bio- 
signals because it promotes electron tunneling from the electroactive center, which is usually buried in a glycoprotein shell of a redox protein [6], to the electrode. The small feature size of the nanoelectrodes also makes their use desirable because the measured currents at the nanoelectrodes exhibit low background noise owing to the minimal charging current that is proportional to the surface area of the electrode. Therefore, nanoscale electrodes are utilized in the low-level signal measurement to improve the signal-to-noise ratio and enhance sensitivity of the electrochemical biosensors.

a)

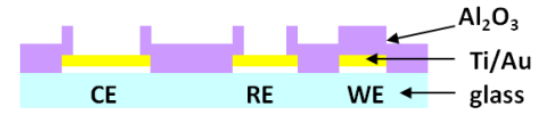

b)

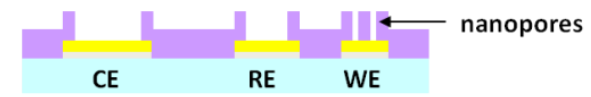

c)

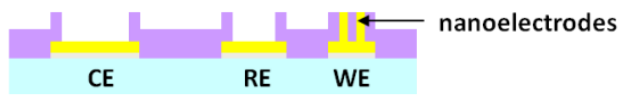

d)

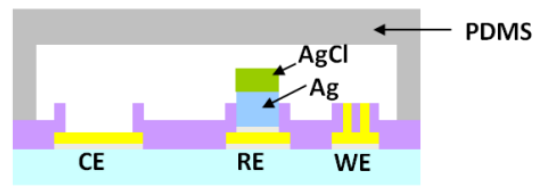

Fig. 1. The fabrication steps used to prepare a nanoelectrode array in a three-electrode cell embedded in a PDMS microchannel. (a) Patterning of the electrodes and the alumina insulating layer using photolithography, e-beam evaporation, and a lift-off method. (b) $I_{2}$-assisted FIB milling and ion beam sculpting of the pore openings. (c) Electrodeposition of $\mathrm{Au}$ into the FIB milling nanopores. (d) $\mathrm{Ag} / \mathrm{AgCl}$ electrode fabrication and assembly with a PDMS microchannel network.

Accordingly, current research activities in the development of innovative electrochemical biosensors have been focusing on the fabrication and characterization of nanoscale electrodes, especially nanoelectrode arrays having the current response which resembles that of individual nanoelectrodes working in parallel. For the design and fabrication of nanoelectrode arrays, the concerns are focused on the inter-electrode distance which should be sufficiently large compared to the electrode radius in order to prevent overlapping of the depletion zones. The choice of materials used for the nanoelectrodes' passivation layer is also crucial such that the films should have high density to prevent any leakage current paths, as well as they should be thick enough to minimize parasitic coupling. In this work, we focus on the fabrication details for the construction of the nanoelectrode arrays used as the working electrodes in the microfluidic three-electrode cells described in the previous work [7]. The fabrication process consists of preparation of dense $\mathrm{Al}_{2} \mathrm{O}_{3}$ films as the insulating layer via electron-beam evaporation, fabrication of a high-aspect-ratio nanoscale pore structure in the insulating layer through $\mathrm{I}_{2}$ gas-assisted focused ion beam (FIB) milling followed by ion beam sculpting, and electrodeposition of $\mathrm{Au}$ to fill the nanopores. Fig. 1 illustrates the fabrication steps used to prepare a nanoelectrode array in a threeelectrode cell, consisting of a working electrode (WE), a reference electrode (RE), and a counter electrode (CE), embedded in a PDMS microchannel network. Cyclic voltammetry (CV) with a standard redox species is performed in the microchannel environment to investigate the electrochemical responses of the prepared electrode cells.

\section{Experimental}

The electrode platform composed of $20-\mathrm{nm} \mathrm{Ti}$ as adhesion layer and 300-nm thick $\mathrm{Au}$ are prepared on a cleaned glass slide using UV lithography, electron-beam evaporation, and liftoff as reported previously [7]. To prepare the electrodes utilized in aqueous solutions, dense films for the insulating sheath are preferred because they are chemically resistant. Besides, the leakage current of the porous insulation may affect the current response when the films are used for the passivation layer of nanoelectrodes. Electron-beam evaporated alumina $\left(\mathrm{Al}_{2} \mathrm{O}_{3}\right)$ is developed as an insulating layer in this work because the fabrication method is compatible with the batch microfabrication process, i.e. the fabrication method does not require high temperature processing. In addition, the $\mathrm{Al}_{2} \mathrm{O}_{3}$ films are found to have higher density $\left(3.1 \mathrm{~g} / \mathrm{cm}^{3}\right.$ [8]) than that of plasma-enhanced chemical vapor deposited (PECVD) $\mathrm{Si}_{3} \mathrm{~N}_{4}\left(2.5 \mathrm{~g} / \mathrm{cm}^{3}\right.$ [9]) which is typically used as the passivation layer for the microelectrodes [10].

The electrode pattern on the glass substrate is processed with UV lithography using 4.5- $\mu \mathrm{m}$ thick $\quad A Z^{\circledR} \quad 9245 \quad$ (Clariant Corporation, Somerville, NJ) photoresist pattern for the subsequent lift-off of $\mathrm{Al}_{2} \mathrm{O}_{3}$ at reference electrodes and counter electrodes. Preceding the deposition of alumina, the substrate temperature is ramped slowly to $180-200{ }^{\circ} \mathrm{C}$ and is then stabilized. Heating the substrate to at least $200{ }^{\circ} \mathrm{C}$ during evaporation is necessary to ensure good adhesion and improved step coverage [11]. The electron beam with the power level of $490 \mathrm{~W}(7 \mathrm{kV}, 70 \mathrm{~mA})$ is used during the deposition. Alumina is deposited at the rate of $3 \AA / s$ until the resulting film thickness 
of $720-800 \mathrm{~nm}$ is obtained. After the photoresist lift-off process, the substrate is then annealed in air at $300^{\circ} \mathrm{C}$ for $4 \mathrm{~h}$ in order to reduce the density of interfacial traps, thus increase the packing density of the alumina sheath for better insulating properties [9, 12]. Fig. 2 shows the fabricated three-electrode cell after patterning the alumina passivation layer over the electrodes on a glass substrate.

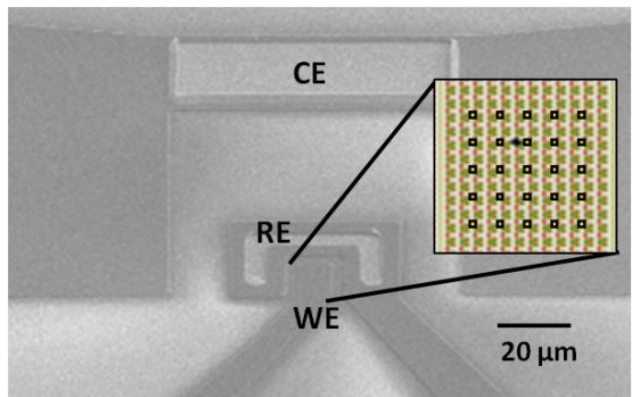

Fig. 2. A SEM image showing the three-electrode cell after patterning the alumina passivation layer over the electrodes. The inset shows the pattern of a nanoelectrode array at the working electrode.

Nanopore arrays can be prepared by creating a high-aspect-ratio pore structure in the insulating layer such that the pores make contact with the underlying $\mathrm{Au}$ layer using the $\mathrm{I}_{2}$ gas-assisted FIB (FEI DB235) enhanced etching technique. $\mathrm{I}_{2}$ gas is directed toward the surface of the sample while alumina is being milled with the ion beam. Sputtered material chemically reacts with the assisting gas to form a volatile compound that can be pumped away for faster etching. The $\mathrm{Ga}^{+}$ion focused beam current is $10 \mathrm{pA}$, and ion beam energy is $30 \mathrm{keV}$. The dwelling time and the overlap between pixels are set to $0.5 \mu$ s and $50 \%$, respectively. A $5 \times 5$ array pattern of $50 \times 50 \mathrm{~nm}$ square windows with 1- $\mu \mathrm{m}$ pore-to-pore spacing is defined at each working electrode area as illustrated in Fig. 2. The milling time has been optimized using test samples to obtain the minimum milling time to expose the buried Au layer. After the focused ion beam milling, the size of the pore window at the surface is typically much larger than the targeted milling pattern size and is also dependent on the milling time. The resulting diameters of the nanopores range between $120-200 \mathrm{~nm}$ as shown in Fig. 3a. Due to a charging effect, the FIB milling process on an insulating substrate needs to be performed at lower magnification. This restriction can affect the resolution of the resulting pattern. It is observed that the actual pore is much larger than the intended pattern size. To reduce the size of the pore tops, we use the ion beam sculpting method [13]. Unfocused ion beam bombardment increases the mobility and diffusivity of the atoms at the surface. As a result, mass transport of the exposed material along the surface promotes partial closing of the pore tops. We use a high speed scanning and low ion beam current to avoid heavy ion etching of the materials. Following the FIB milling of nanpores, rastering of the ion beam over the whole array using ion beam energy of $30 \mathrm{keV}, 10 \mathrm{pA}$ ion beam current, and scanning time of $0.724 \mathrm{~s}$ per scan for $15-20$ min reduces the initial pore opening diameters to $60-120 \mathrm{~nm}$. Fig. $3 \mathrm{~b}$ shows an SEM image of the nanopore array after the ion beam sculpting

process.
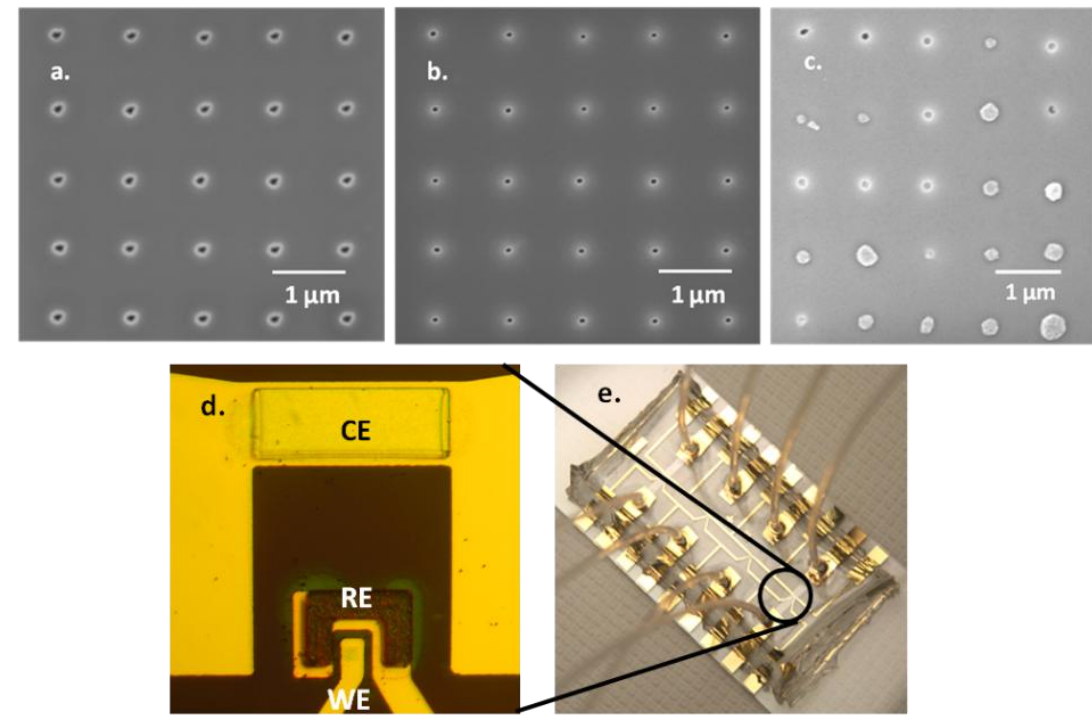

Fig. 3. Fabrication results. (a) A nanopore array after the $I_{2}$ gas-assisted FIB milling and (b) after the subsequent ion beam sculpting process. (c) A nanoelectrode array after the Au electrodeposition process. (d) The complete three-electrode cell. (e) The flow-chip after the bonding process. 
The pores are then filled by dc electrodeposition of $\mathrm{Au}$ at $-0.8 \mathrm{~V}$ for approximately $2 \mathrm{~h}$ in Orotemp 24 gold plating solution (Technic Inc., Cranston, RI, USA), using a platinum wire as an anode. Fig. 3c shows the shows a SEM image of a nanopore array after the filling of $\mathrm{Au}$ via electrodeposition. The fabrication of the reference $\mathrm{Ag} / \mathrm{AgCl}$ electrodes, the PDMS microchannels, and the chip assembly process are reported previously [10]. Fig. 3d shows the optical microscope image of the complete three-electrode cell. Fig. $3 e$ shows the image of the flow-chip after the bonding process. Electrochemical performance of the nanoelectrode array in a microchannel environment is investigated by cyclic voltammetry using the analyte solution of $5 \mathrm{mM}$ $\mathrm{Ru}\left(\mathrm{NH}_{3}\right)_{6} \mathrm{Cl}_{3}$ in $25 \mathrm{mM} \mathrm{Na}_{2} \mathrm{HPO}_{4}$ and $25 \mathrm{mM}$ $\mathrm{NaCl}$ (pH 7.0).

Normalized current density $\left(\times 10^{6} \mathrm{~A} \cdot \mathrm{cm} \cdot \mathrm{mol}^{-1}\right)$

a.
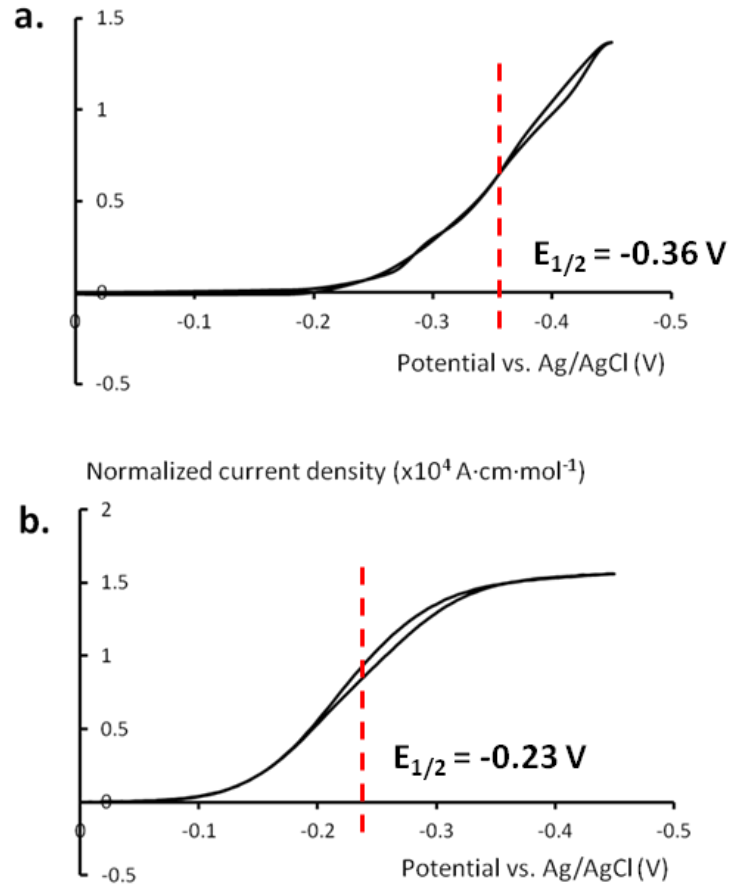

Fig. 4. CV results showing (a) the response of a nanoelectrode array and (b) the response of a microelectrode with the diameter of $3 \mu \mathrm{m}$.

\section{Results and Discussion}

Fig. 4 shows the $\mathrm{CV}$ responses at the scan rate of $100 \mathrm{mV} / \mathrm{s}$ of the nanoelectrode array and that of a $\mathrm{Au}$ microelectrode with the diameter of 3 $\mu \mathrm{m}$. As observed from the voltammograms, the current density obtained at the nanoelectrode arrays is increased by two orders of magnitude compared to that obtained from the microelectrode. The steady-state limiting current plateau is not as well-defined. The effects of high mass transport rate due to the reduced depletion layer thickness and the high electric field generated near the electrode surface are accounted for the significant deviation from the classical theory of diffusionlimited current flow. In addition, in the case of the nanoelectrodes, the half-wave potential is shifted to the negative direction. This shift is due to the high current density which alters the mass transport from the diffusion-controlled process to the charge-transfer-controlled process [3]. Hence, the observed characteristics of the $\mathrm{CV}$ responses at the nanoelectrode arrays verify the performance of the nanoscale electrodes such that extraction of low-level signals from electrochemical biosensors can be achieved.

\section{References}

[1] J.D. Norton, H.S. White, S.W. Feldberg, Journal of Physical Chemistry 94, 6772-6780 (1990); doi: 10.1021/j100380a044

[2] R. He, S. Chen, F. yang, and B. Wu, The Journal of Physical Chemistry B 110, 3262-3270 (2006); doi: 10.1021/jp060084

[3] J. Chen and K. Aoki, Electrochemistry Communications 4, 24-29 (2002); doi: 10.1016/S1388-2481(01)00256-9

[4] A.J. Bard and L.R. Faulkner, In Electrochemical Methods: Fundamentals and Application. New York, NY: John Wiley \& Sons, p. 208 (1980).

[5] R.M. Wightman and D.O. Wipf, In Electroanalytical Chemistry, ed. A.J. Bard, Marcel Dekker, New York, vol. 15, p. 267 (1989).

[6] A. Heller, The Journal of Physical Chemistry 96, 3579-3587 (1992); doi: 10.1021/j100188a007

[7] N. Triroj, P. Jaroenapibal, H. Shi, J.I. Yeh, and R. Beresford, Biosensors and Bioelectronics 26, 2927-2933 (2011); doi: 10.1016/j.bios.2010.11.039

[8] W.M. Gosney and R.S. Miller, Thin Solid Films 14, 255-266 (1972); doi: 10.1016/00406090(72)90427-0

[9] M. Arps and A. Markwitz, Journal of Vacuum Science and Technology A 15, 1864-1873 (1997); doi: 10.1116/1.580653

[10] N. Triroj, M.A. Lapierre-Devlin, S.O. Kelley, and R. Beresford, IEEE Sensors Journal 6,1395-1402 (2006); doi: 10.1109/JSEN.2006.884444

[11] B. Lewis, Microelectronics Reliability 3, 109-120 (1964); doi: 10.1016/0026-2714(64)90245-8

[12] S. Shuzhen, C. Lei, H. Haihong, Y. Kui, F Zhengxiu, and S. Jianda, Applied Surface Science 242, 437-442 (2005); doi: 10.1016/j.apsusc.2004.09.047

[13] J. Li, D. Stein, C. McMullan, D. Branton, M.J. Aziz, and J.A. Golovchenko, Nature 412, 166-169 (2001); doi: 10.1038/35084037 